\title{
Evaluation of Haemodynamic Changes of Proximal Arterial Occlusion in the Lower Extremities by Spectrum Doppler Ultrasonography
}

\author{
Z Wang, W Shi, J Zhou, G Sui, H Wang
}

\begin{abstract}
Objective: The objective is to analyse the changes of haemodynamic and Doppler ultrasonography spectrum in the proximal arterial occlusion of lower extremities, and to explore the value of spectrum Doppler ultrasonography in the diagnosis of lower extremity arterial occlusive disease.

Methods: Thirty-four patients (thirty-nine occlusion arteries) were examined with two-dimensional ultrasound combined with colour Doppler flow imaging (CDFI), and the results were compared.

Result: The waveform shape in the artery proximal to the occlusion shows that blood flow velocity becomes slower, and diastolic reverse wave slowly disappears. Systolic waveform records a steep upstroke, a slow downstroke and a shoulder on the downstroke. The appearance of the shoulder on the downstroke is earlier than the diastolic upward wave. The differences of peak systolic velocity, acceleration time, deceleration time, systolic acceleration, deceleration, diastolic reverse flow velocity, systolic acceleration time/systolic duration and systolic deceleration time/systolic duration in the proximal occlusion segment compared with the control group are statistically significant $(\mathrm{p}<0.05)$.

Conclusion: Artery occlusion can lead to haemodynamic response and spectrum waveform change. Timely discovery of the changes of the spectrum can be of clinical significance in the diagnosis of lower extremity arterial occlusive disease.
\end{abstract}

Keywords: Arterial occlusive disease, Doppler spectrum, lower limb extremity

WIMJ Open 2015; 2 (2): 77

\section{INTRODUCTION}

Lower limb extremity arterial occlusive disease is a major health problem in developed and developing countries, where lifestyle factors such as smoking (1) and drinking (2) can accelerate the progression of the disease. Lower extremity arterial occlusive disease, which mainly affects those aged above 50 years, has become a common peripheral vascular disease with high morbidity in patients with high blood pressure (3), high cholesterol $(4,5)$ and diabetes $(6)$.

For a long time, arterial X-ray digital subtraction angiography (DSA) has been the gold standard for peripheral arterial occlusive disease diagnosis, but DSA has the disadvantages of being invasive and expensive (7), which limits its clinical application. With the development of colour Doppler ultrasound, colour Doppler diagnosis for lower extremity arterial disease has become mainstream $(8,9)$. In recent years, many scholars have conducted research on the occluded artery spectrum, which is mostly about the distal

From: Department of Ultrasonography, China-Japan Union Hospital of Jilin University, Changchun 130033, China.

Correspondence: Dr H Wang, Department of Ultrasonography, China-Japan Union Hospital of Jilin University, No. 126 Xiantai Street, Changchun 130033, China. E-mail: huiwangdoc@126.com segment of occlusion (10). There is little research on the spectrum of proximal arterial occlusion. We used spectrum Doppler ultrasonography to evaluate the haemodynamic changes of an artery proximal to an occlusion to observe the changes of spectrum waveform shapes, in order to provide a new reference of lower limb arterial occlusion disease.

\section{SUBJECTS AND METHODS}

Thirty-four patients with lower limb arterial occlusion disease were enrolled in this study that took place in the China-Japan Union Hospital of Jilin University from January 2012 to January 2013. There were 39 diseased lower limbs. The occlusion sections were located in the superficial femoral artery and/or popliteal artery, and there was no significant stenosis proximal to the point of occlusion. In the 34 patients, there were 28 males and six females, age range 4478 years old. They were all diagnosed with lower limb occlusive artery disease by computed tomography angiography (CTA). The control group consisted of 10 males and nine females who did not smoke, did not have lower limb arteriosclerosis, vascular malformation, stenosis, or arterial expansion. Blood pressure, blood glucose and lipid levels were in the normal range. This study was conducted in accordance with the Declaration of Helsinki and was 
approved by the Ethics Committee of China-Japan Union Hospital of Jilin University. Written informed consent was obtained from all participants.

\section{Lower extremity duplex scanning}

The device used was an IU22 colour Doppler ultrasonic diagnostic instrument from Philips (Amsterdam, The Netherlands). Linear array transducer frequency ranged from 5-12 $\mathrm{MH}$, sample volume size was $1-2 \mathrm{~mm}$ and the angle of intonation between the Doppler ultrasound beam and blood flow was in the lower $60^{\circ}$ angle.

The patient should be relaxed and lying in a supine position with the lower limb fully exposed, the leg rotated outward and the knee gently flexed. The femoral artery from the level of the groin, the superficial femoral artery, and then the profunda femoris artery were scanned. The popliteal artery, with the patient in a prone position, as well as the anterior tibial artery, posterior tibial artery, and the dorsalis pedis artery, while the patient sat with the knee flexed, were also scanned. The transducer was moved along the blood vessel, from a proximal to a distal position. We used both the transverse section and longitudinal section to compare and contrast the scans.

\section{Statistical analysis}

The experimental data are presented as a mean \pm standard deviation. Data were analysed using the $T$ test with the Statistical Package for Social Sciences (SPSS). Statistical significance was established with $p<0.05$.

\section{RESULTS}

\section{Proximal waveform changes due to artery occlusion}

Waveform shapes are obvious when the proximal artery is occluded (11). Blood flow velocity becomes significantly slower and diastolic reverse waves become slower or monophasic. A disordered waveform shape has a spectral window that becomes smaller or disappears, spectral broadening, a systolic upward steep slope and a shortened acceleration time. Furthermore, the acceleration increases, the systolic downward slope flattens, there is a characteristic shoulder on the systolic downward slope, the deceleration time extends and deceleration becomes smaller. The closer the sampling volume position is to the occlusion area, the more significantly the waveform shapes change (12). The characteristic shoulder on the systolic downward slope appears before disappearance of the diastolic reverse wave.

The sampling volume was placed at the position of 2 $\mathrm{cm}$ above the occlusion. The waveform shape was observed and compared to the control group. There were 37 arteries that had the systolic peak on the downward slope out of a total of 39 diseased arteries, which is $94.9 \%$. There were 33 arteries that had the diastolic second and third wave disappear [84.6\%, 33/39] (13). All disappearances of the diastolic reverse waves come along with the systolic peak on the downward slope.

\section{Changes of velocity}

The peak systolic velocity (PSV) is $78.5 \pm 32.6 \mathrm{~cm} / \mathrm{s}$, which is distinctly low compared with the control group $[p<0.05]$ (14). The diastolic reverse flow velocity (DRV) is $1.7 \pm 4.3$ $\mathrm{cm} / \mathrm{s}$, which is evidently low compared with the control group [ $p<0.05]$ (Table 1).

Table 1: Peak systolic velocity (PSV) and diastolic reverse flow velocity (DRV) $(x \pm \mathrm{s})$ in the same position of the occlusion group and control group

\begin{tabular}{lccc}
\hline Group & $\mathbf{n}$ & PSV $(\mathbf{c m} / \mathbf{s})$ & DRV $(\mathbf{c m} / \mathbf{s})$ \\
\hline Occlusion & 39 & $78.5 \pm 32.6^{*}$ & $1.7 \pm 4.3^{*}$ \\
Control & 38 & $112.4 \pm 18.5$ & $12.6 \pm 2.2$ \\
\hline
\end{tabular}

${ }^{*} p<0.05, v s$ the control group

\section{Changes of systolic upstroke}

Systolic acceleration time (AT) is $68.3 \pm 19.8 \mathrm{~ms}$, which is shorter compared with the control group $(p<0.05)$. The systolic acceleration (AC) is $1752.6 \pm 1075.7 \mathrm{~cm} / \mathrm{s}^{2}$, which is clearly larger than the control group $(p<0.05)$. The systolic acceleration time/systolic duration (AT/ST) is $0.23 \pm 0.06$, which is obviously small compared with the control group $[p$ $<0.05$ ] (Table 2).

Table 2: Acceleration time (AT), systolic acceleration (AC) and systolic acceleration time/systolic duration (AT/ST) $(x \pm \mathrm{s})$ in the same position of the occlusion group and control group

\begin{tabular}{lcccc}
\hline Group & $\mathbf{n}$ & AT $(\mathbf{m s})$ & $\mathbf{A C}\left(\mathbf{c m} / \mathbf{s}^{\mathbf{2}}\right)$ & $\mathbf{A T} / \mathbf{S T}$ \\
\hline Occlusion & 39 & $68.3 \pm 19.8^{*}$ & $1752.6 \pm 1075.7^{*}$ & $0.23 \pm 0.06^{*}$ \\
Control & 38 & $112.4 \pm 18.5$ & $816.2 \pm 129.3$ & $0.44 \pm 0.05^{*}$ \\
\hline
\end{tabular}

${ }^{*} p<0.05, v s$ the control group

\section{Changes of systolic downstroke}

The deceleration time (DT) is $219.2 \pm 24.6 \mathrm{~ms}$, long compared with the control group $(p<0.05)$. The deceleration (DC) is $342.3 \pm 73.4 \mathrm{~cm} / \mathrm{s}^{2}$, which is obviously smaller when compared with the control group $(p<0.05)$. The systolic deceleration time/systolic duration (DT/ST) of $0.77 \pm 0.06$ is larger compared with the control group $[p<0.01]$ (Table 3 ).

Table 3: Deceleration time (DT), deceleration (DC) and systolic deceleration time/systolic duration (DT/ST) $(x \pm \mathrm{s})$ in the same position of the occlusion group and control group

\begin{tabular}{lcccc}
\hline Group & $\mathbf{n}$ & DT $(\mathbf{m s})$ & DC $\left(\mathbf{c m} / \mathbf{s}^{2}\right)$ & DT/ST \\
\hline Occlusion & 39 & $219.2 \pm 24.6^{*}$ & $342.3 \pm 73.4^{*}$ & $0.77 \pm 0.06^{*}$ \\
Control & 38 & $140.7 \pm 19.9$ & $651.3 \pm 168.5$ & $0.56 \pm 0.08$ \\
\hline
\end{tabular}

${ }^{*} p<0.05, v s$ the control group

\section{DISCUSSION}

The normal spectral Doppler display recorded from a lower limb extremity artery is a triphasic flow pattern with a clear spectral window (Fig. 1). The first phase wave is the sharp 


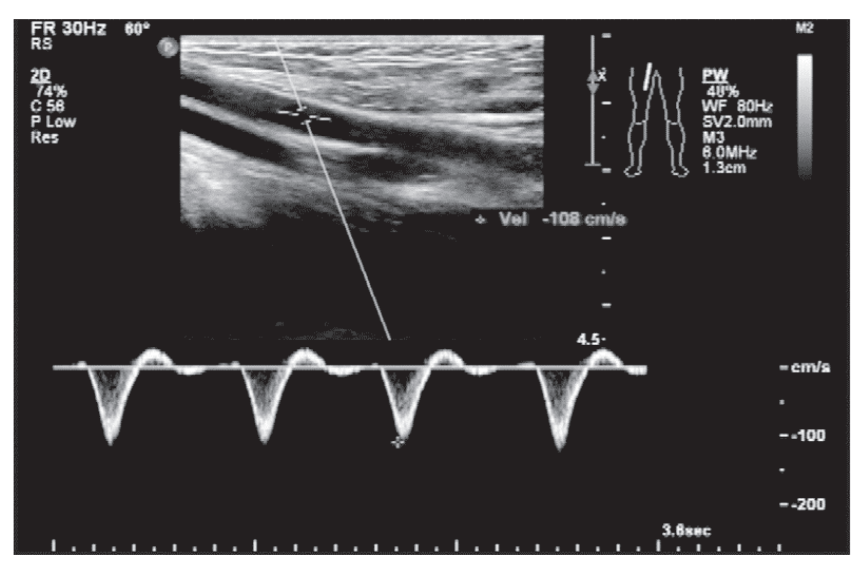

Fig. 1: A normal triphasic waveform.

forward wave, which is caused by the positive blood flow of the cardiac systolic ejection. This is characterized by rapid rise and fall strokes. The second phase consists of a reverse wave, which is caused by an early diastolic aortic valve closure and a peripheral vascular elasticity retraction. The third phase wave is caused by arterial elasticity leading to the retraction of blood flow to the distal limb again in late diastole. The presence of normal triphasic flow is an indicator that the lower segment of the artery before the large branches has not been occluded or severely stenosed (15).

The waveform shapes in the area proximal to an occlusion lose the characteristic triphasic flow pattern in these 39 cases. Instead, there are either monophasic or variant triphasic flow patterns. The upstroke of the first wave became steep, which is drastically different compared with the control group $[p<0.05]$ (16). The result may be relevant to the variety of conditions of the area proximal to an occlusion (17). If there is no occlusion or severe stenosis, no large branches in the region proximal to an occlusion, then there is no haemodynamic impact. If the waveform shape in the area proximal to an occlusion looks like a hill then this is characteristic of a distal occlusion (Fig. 2). The ejection time would be prolonged if the arterial branches were above the occlusion, where blood can flow away. The ejection time

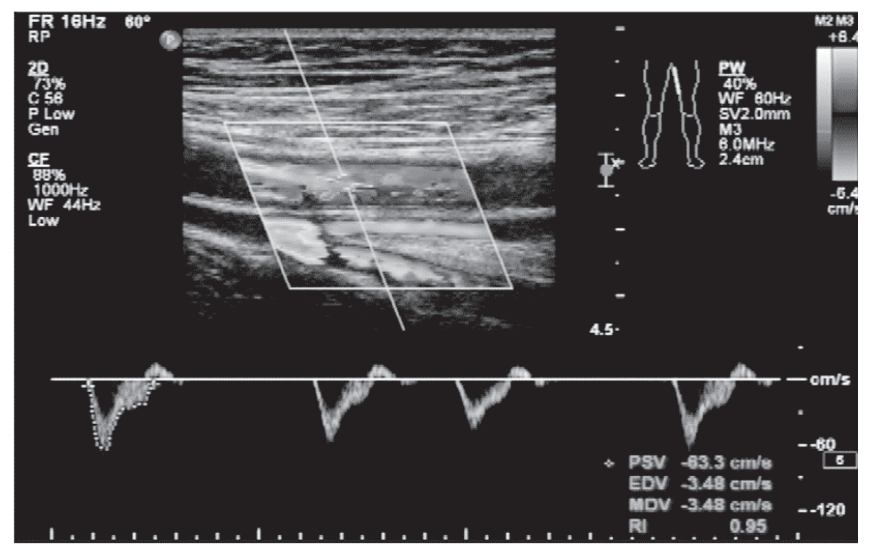

Fig. 2: The waveform of distal occlusion. would be shortened if no arterial branches existed above the occlusion, where blood returns.

The downstroke shape of the first wave is flat. This downstroke is at the phase of the slow ejection period of the cardiac cycle. The resistance is as large as the distal occlusion, which can offset the part of the cardiac systolic blood pressure. Blood potential energy in the blood vessels falls slowly, so the downstroke shape of the first wave is flat. The result is distinctly different compared to the control group ( $p$ $<0.05)$.

On the spectrum, the diastolic reverse flow velocity measured at the peak of the early diastolic reverse wave can reflect the vessel compliance. Diastolic reverse flow velocity is relevant to the bed resistance of distal blood vessels. Vascular bed resistance increases, and elastic retraction forces decrease when lower limb extremity arteries are occluded. This leads to DRV slow down or disappearance (18) [Fig. 3].

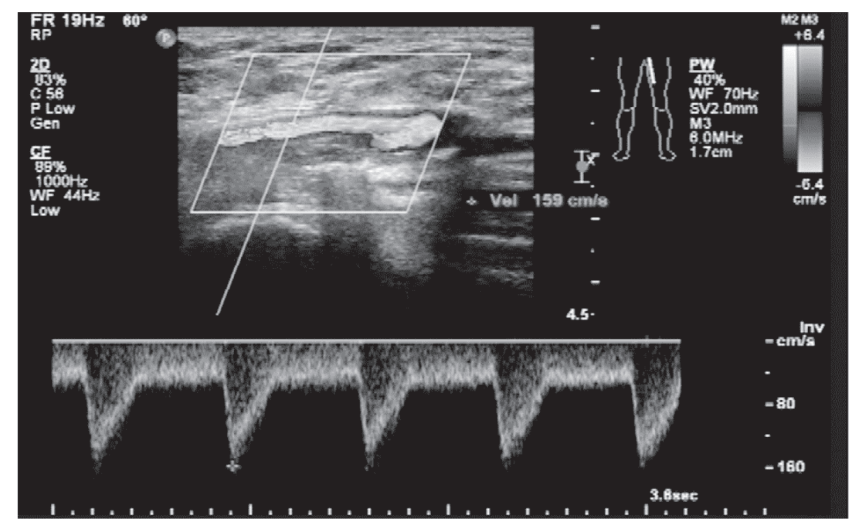

Fig. 3: The second wave disappears.

In this study, the characteristic shoulder on the systolic downstroke (Fig. 4) appears in the area proximal to the occlusion of the arteries of 37 cases, and not in the other two cases. The time in which the shoulder appears is $88 \pm 2 \mathrm{~ms}$. The two cases that have no shoulders on the systolic downstroke are blocked at the bifurcation of the common femoral artery and superficial femoral artery. In these two cases, the sample volume is located proximal to the origin of the profunda femoris artery. Blood can flow through the profunda femoris artery, which broadens, so the increase of the distal resistance is not obvious. The characteristic shoulder on the systolic downstroke does not appear in the control group. This study's results show that the appearance time of the shoulder on the systolic downstroke coincides with collateral vessels that open proximal to the occlusion (19). When collateral vessels open proximal to the occlusion, the appearance time of the shoulder on the systolic downstroke becomes shorter or ceases to exist. But there is no clear research between the number, pipe diameter, velocity of collateral vessels, or the appearance time of the shoulder on the systolic downstroke. This will be discussed in subsequent research. 


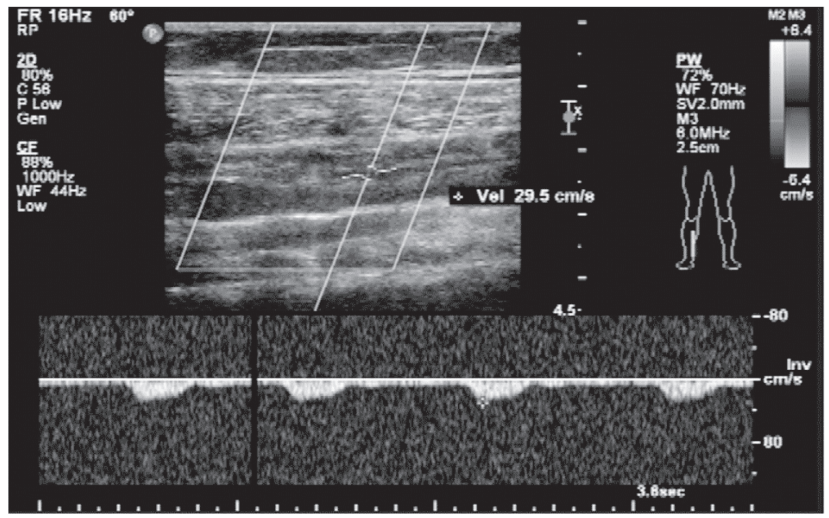

Fig. 4: Characteristic shoulder on the systolic downstroke.

At the same time, we found that the rate of the characteristic shoulder appearance on the systolic downstroke is $94.9 \%$; the rate of the second and the third wave's disappearance is $84.6 \%$. When observing the waveform shape from the upper to the lower area proximal to the occlusion of the artery, the shoulder appears before the second wave disappears. Through the multi-point measurement proximal to the occlusion, we found that the changes of the waveform shape are gradual. In our opinion, the characteristic shoulder on the systolic downstroke suggesting distal disease is more sensitive than the disappearance of the second wave.

The occlusion segment that is located in the blind scan area, a deep location, or covered with dressing, cannot be detected easily. We can suspect the distal artery through the proximal waveform shape, which makes up for the limitation of 2D and colour Doppler ultrasound, and which improves the diagnostic rate. Through quantitative analysis of waveform shape proximal to an occlusion, and the observation of haemodynamic changes, a new reference to clinical diagnosis can be made (20).

\section{CONFLICT OF INTEREST}

All authors have no conflict of interest regarding this paper.

\section{REFERENCES}

1. Vicente I, Alves A, Pereira A, Roncon de Albuquerque R. Evaluation of hemodynamic changes in Buerger's disease patients. Acta Med Port 2012; 25: 156-64.

2. Wakabayashi I, Sotoda Y. Alcohol drinking and peripheral arterial disease of lower extremity. Nihon Arukoru Yakubutsu Igakkai Zasshi 2014; 49: 13-27.

3. He M, Qin X, Cui Y, Cai Y, Sun L, Xu X et al. Prevalence of unrecognized lower extremity peripheral arterial disease and the associated factors in Chinese hypertensive adults. Am J Cardiol 2012; 110: $1692-8$.

4. Korneva VA, Kyznetsova TIu, Mandel'shtam MIu, Konstantinov VO, Vasil'ev VB. The clinical manifestations of atherosclerosis in familial hypercholesterolemia. Ter Arkh 2014; 86: 18-22.

5. Shaalan WE, French-Sherry E, Castilla M, Lozanski L, Bassiouny HS. Reliability of common femoral artery hemodynamics in assessing the severity of aortoiliac inflow disease. J Vasc Surg 2003; 37: 960-9.
6. Yu LB, Shen Y, Li LX, Tu YF, Liu F, Bao YQ et al. Detection rates of atherosclerosis by carotid versus lower limb ultrasonography in newly diagnosed type 2 diabetics. Zhonghua Yi Xue Za Zhi 2013; 93: 21435.

7. Pomposelli F. Arterial imaging in patients with lower extremity ischemia and diabetes mellitus. J Vasc Surg 2010; 52: 81S-91S.

8. Khan SZ, Khan MA, Bradley B, Dayal R, McKinsey JF, Morrissey NJ. Utility of duplex ultrasound in detecting and grading de novo femoropopliteal lesions. J Vasc Surg 2011; 54: 1067-73.

9. Gabriel M, Pawlaczyk K, Szajkowski R, Stefaniak K, Dzieciuchowicz L, Krasiński Z. The use of duplex ultrasound arterial mapping (DUAM) and preoperative diagnostics in patients with atherosclerotic ischaemia of lower extremities. Pol Przegl Chir 2012; 84: 276-84.

10. Sukharev II, Guch AA, Novosad EM, Vlaĭkov GG. Collateral compensation of blood flow and hemodynamics of the lower extremities in atheroselerotic occlusion of abdominal aortal. Kiln Khir 2001; 12: 14-6.

11. Styczynski G, Szmigielski C, Leszczynski J, Kuch-Wocial A, Szulc M. Abdominal aortic Doppler waveform in patients with aorto-iliac disease. Eur J Vasc Endovasc Surg 2010; 39: 714-8.

12. Kamran H, Salciccioli L, Prudhvi K, Bastien C, Berman H, Sharma A et al. Comparison of hyperemic changes in carotid-radial pulse wave velocity by upper and lower arm cuff occlusion. Angiology 2011; 62: 409-14.

13. Tsai CY, Chu SY, Wen YW, Hsu LA, Chen CC, Peng SH et al. The value of Doppler waveform analysis in predicting major lower extremity amputation among dialysis patients treated for diabetic foot ulcers. Diabetes Res Clin Pract 2013; 100: 181-8.

14. Okada M, Kitano I, Sugimoto T, Ijiri Y. Functional diagnosis with color ultrasound in occlusion of peripheral arteries. Kongressbd Dtsch Ges Chir Kongr 2002; 119: 623-6.

15. Legemate DA, Teeuwen C, Hoeneveld H, Ackerstaff RG, Eikelboom BC. Spectral analysis criteria in duplex scanning of aortoiliac and femoropopliteal arterial disease. Ultrasound Med Biol 1991; 17: 76976.

16. Lee HG, Yum MK. Fourier transformation of arterial Doppler waveforms of the lower extremity. J Clin Ultrasound 2004; 32: 277-85.

17. Wen XR, Lü XF, Liu CC, Luo Y, Chen DW, Wang C et al. The ultrasound image characteristics of lower extremities arteries in diabetic foot. Sichuan Da Xue Xue Bao Yi Xue Ban 2012; 43: 739-42.

18. Styczynski G, Szmigielski C, Leszczynski J, Abramczyk P, KuchWocial A, Szulc M. Descending aortic Doppler flow pattern in patients with proximal peripheral artery disease. Am J Cardiol 2009; 103: 1774 6.

19. Valecchi D, Bacci D, Gulisano M, Conti AA, Sibilio M, Lipoma M et al. Evaluation of the pattern of proximal and distal occlusion and collateral circulation of lower limb arteries using combined contrast arteriography and color Doppler ecography. Ital J Anat Embryol 2009; 114: $121-7$.

20. Seige M, Podhaisky H, Hänsgen K. Arterial vascular diagnosis by Doppler pulse curve analysis of the first dorsal metatarsal artery. Z Gesamte Inn Med 1993; 48: 484-9.

Received 15 Oct 2014

Accepted 24 Oct 2014

Published 12 Jun 2015

Online: http://www.mona.uwi.edu/wimjopen/article/1632

(C) Wang et al 2015.

This is an open access article made freely available under Creative Commons Attribution 4.0 International (CC BY 4.0). Users are free to share, copy and adapt this work as long as the copyright holder (author) is appropriately and correctly credited. See http://creativecommons.org/ licences/by/4.0/deed.en_us for more information. 